\title{
Evaluation of Wearable Gyroscope and Accelerometer Sensor (PocketIMU2) during Walking and Sit-to-Stand Motions
}

\author{
Qi An ${ }^{1}$, Yuki Ishikawa ${ }^{1}$, Junki Nakagawa ${ }^{1}$, Atsushi Kuroda ${ }^{2}$, \\ Hiroyuki Oka ${ }^{3}$, Hiroshi Yamakawa ${ }^{1}$, Atsushi Yamashita ${ }^{1}$ and Hajime Asama ${ }^{1}$
}

\begin{abstract}
Recently healthcare of the elderly people has become a serious issue in medical and rehabilitation areas. In order to know their functional mobility and provide sufficient medical treatment, it is important to measure their body state precisely and objectively. Therefore we developed a wearable and wireless sensor of gyroscope and accelerometer (PocketIMU2) as an easy and precise measurement of human motions. In the sensor, we employed a small and high accurate LiNbO3 crystal to achieve joint angle computation with simple integration of angular velocity. In the current paper, we evaluate the accuracy of the sensor in two important basic motion, such as a walking and sit-to-stand motions. Computed joint angles of ankle, knee, and hip are compared to the reference data measured from a optical motion capture system in term of coefficients of correlation and root mean square error. As a result, coefficient of correlation showed very high value for all joint angles, and root mean square error was adequately small. This strongly supports the usage of our developed gyroscope and accelerometer sensor for monitoring human body movement for medical usage.
\end{abstract}

\section{INTRODUCTION}

These days an aging society has become a major social issue in healthcare and wealth areas. Many elderly people have suffered from their decreased physical ability or changed structure of the musculoskeletal system. For example, when elderly people get knee osteoarthritis or back pain, their activities of daily living (ADL) is known to be decreased [1]. These situations have brought many problems to our society, such as lower productivity or increased social security cost.

In order to improve the situation, it is important to know their body state and provide them a sufficient diagnosis or treatment. For instance, there is a widely used important clinical tool, called the timed up and go test (TUG) which assess functional balance and mobility [2]. Traditionally, the test is scored by manually recording the time taken to rise out of a standardized chair, walk, turn and sit back down in the chair. Therefore, it causes ambiguous time measurement or it cannot measure dynamic state.

Recent sensor technology has enabled us to measure human body sate precisely. For example, optical motion capture systems, force plates, electric goniometer or combinations of those sensors have been suggested as an effective measurement tools. However, costs of those systems usually are expensive or have space restriction. Another research

\footnotetext{
1 Q. An, Y. Ishikawa, J. Nakagawa, H. Oka, A. Yamashita, H. Asama are with Faculty of Precision Engineering, Department of Engineering, The University of Tokyo, Tokyo, Japan (e-mail: anqi@robot.t.u-tokyo.ac.jp)

2 A. Kuroda is with gsport, Inc., Tokyo, Japan

3 H. Oka is with The 22nd Century Medical and Research Center, Graduated School of Medicine, The University of Tokyo, Tokyo, Japan
}

has suggested using Kinect [Microsft Corp.] [3], but its accuracy is comparatively low or it needs careful settings of their location. Thus, they can be used in a well organized laboratory or massive healthcare facilities.

Therefore in this study, we focus on a gyroscope sensor due to its reduced size, lower cost, or unconstrained space. Using the gyroscope sensor, it would be easier to measure human body movement even in a smaller healthcare facilities. However, it is widely known that there is accumulated error (drift) to calculate joint angles from integration of angular velocities.

Previously we developed the wearable gyroscope and accelerometer sensor (PocketIMU). Ishigaki, N. et al. evaluated gait motions of the elderly people (divided into stable and unstable groups) and they found significant less pelvic movement in the unstable group compared to the stable group [4].

Related to a gyroscope sensor, Sagawa, K. et al. successfully recorded human pitching motion with modification of the recorded data by assuming the fixed start and end postures [5]. They could record very quick motion, but their assumption cannot be applicable to continuous daily motions. Another research by Watanabe, T. et al. proposed usage of the Kalman filter, low pass filter to compute joint angles and stride length in human gait [6]. However, those filtering processes might cause delay of data acquisition, or parameter tuning will be necessary.

In this paper, we suggest a new wearable gyroscope and accelerometer sensor with a new piezo element $(\mathrm{LiNbO} 3)$ to achieve a high accuracy, called PocketIMU2. Using the new piezo element, manufacturing cost and size of our sensor has become one-fifth to one-tenth. Also, this sensor realized wireless data recording by bluetooth 2.0 connection to enable users of the sensor to move without paying attention to the wire. In addition, accompanied recording software (described in the later section) is improved to record and display recorded data from several sensors at the same time.

Therefore our objectives in this study are to test the accuracy of our developed gyroscope sensor without any filters. In order to verify their accuracy for medical usage, we employed two fundamental motions which are important for medical usage, such as walking, sit-to-stand, and stand-to-sit, are employed. 


\section{METHODS}

\section{A. Methods Overview}

In the current study, three gyroscope and accelerometer sensors were used to measure joint angles: hip, knee and ankle joints. In human basic daily motions, such as walking, sit-to-stand, and stand-to-sit motions, the accuracy of our developed sensors was evaluated in comparison with body positions measured by an optical motion capture system. For evaluation, coefficients of correlation and root square mean error were calculated.

\section{B. Detailed Configuration of PocketIMU2}

Figure 1-(A) shows external aspect of the developed gyroscope and accelerometer sensor and its three axes. Angular velocity around each axis can be obtained: roll (x-axis), pitch (y-axis), and yaw (z-axis). In addition, acceleration is recorded along each axis.

Inside the sensor (PocketIMU2), LiNb03 Crystal [Tamagawa Seiki Co., Ltd] was used for low noise high precision measuring. LiNbO3 Crystal enabled high accuracy measument by its higher electro-mechanical conversion efficacy and low magnification of amplification ration of its signal processing circuit. Figure 2 shows response characteristic of LiNbO3 Crystal compared to previous gyro element used in the PocketIMU. The graph depicts the voltage change according to the small input angular velocity, such as \pm 0.1 $\mathrm{deg} / \mathrm{s}$, (the bottom purple line); it suggests that new LiNbO3 crystal (the middle blue line) shows much less noise than the previous gyro (top red line) even with the small change of angular velocity.

Size of the sensors is 42 (width) $\times 48$ (depth) $\times 31$ (height) $\mathrm{mm}$ respectively, and their weight is about 0.050

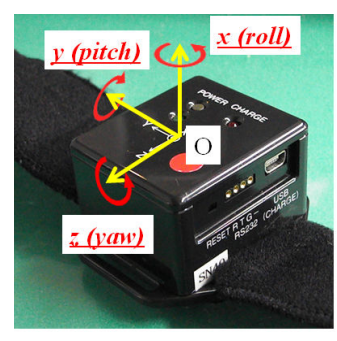

(A)

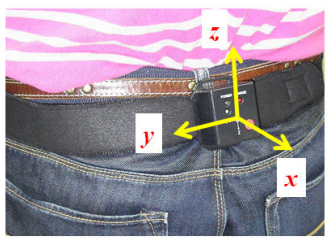

(B)

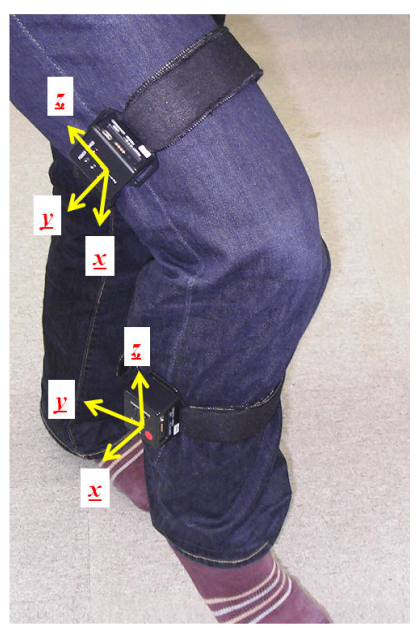

(C)
Fig. 1. Pictures show the developed software used for our experiment. (A) Outer appearance of our sensor. Angular velocities and acceleration are obtained around and along each axis. (B), (C) Location of our sensors to be attached with a participant.

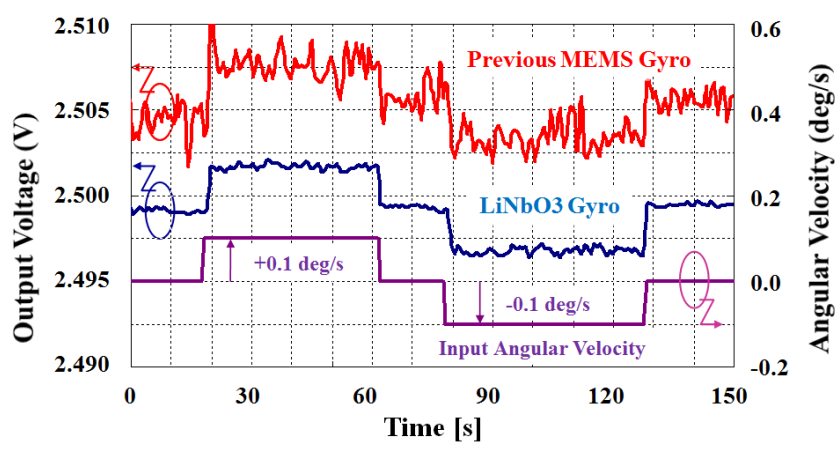

Fig. 2. Above graph shows output comparison between previous MEMS gyro and new LiBNO3 gyro. It shows their output voltage according input angular velocity.

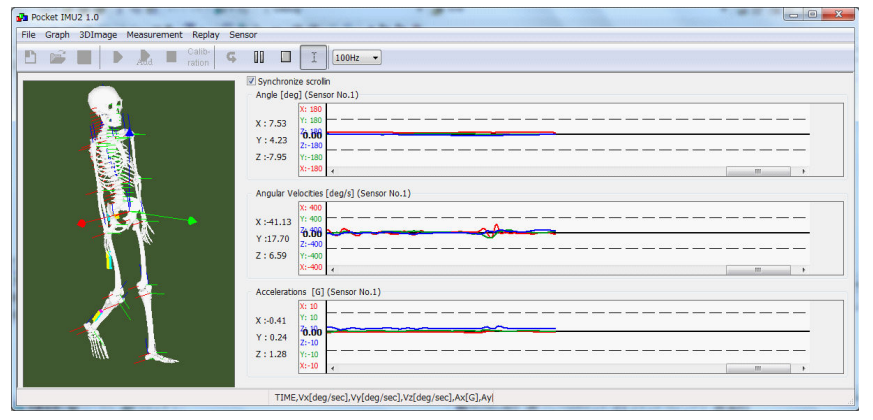

Fig. 3. Display of our developed recording software. The right side of the software shows recorded data from sensors and the left side depicts movement of each joint with the musculoskeletal model.

$\mathrm{Kg}$. It contains a Li-Io rechargeable battery, and its maximum continuous operation time is $60-90 \mathrm{~min}$. Recorded data can be sent to a computer through the bluetooth 2.0 wireless connection or through the RS-232 wired connection. Using bluetooth, range of data recording is $30-100 \mathrm{~m}$ depending on the environment. Detailed data sheet is shown in [7].

Additionally, there is a software accompanied with the developed sensor. Figure 3 shows a screenshot of the software. On the right part of the software, it displays calculated joint angles, angular velocities, and acceleration for each axis. At the same time, it depicts musculoskeletal model with joint movement based on on-line recording. This feature is very useful for medical or rehabilitation usage; medical doctor or physical therapist can monitor and understand movement of patients visually.

\section{Experimental Setup}

1) Setup of PocketIMU: Figure 1-(B), (C) indicate body locations for three sensors to be attached. One sensor was attached to a back side of a pelvic of a participant, another sensor was located on a middle of femoral area, and the other one was placed on a middle of their lower leg. Three angular velocities (roll, pitch and yaw) around each axis were obtained from our sensor.

An elasticized band was used to attach each sensor to a subject body. The band could tighten up the body segment 
with a strong hook and loop fastener in order to fix the positions of sensors. Although the developed sensors could send their obtained data through a bluetooth wireless connection, we used the RS-232 wired connection in this study to synchronize data recording of motion capture system and gyroscope sensors.

Calibration was required to decide the initial axes of the sensor. During the calibration period (7-10 s), subjects were asked to stand or sit still before performing motions.

2) Setup of Optical Motion Capture System: In order to measure reference body positions during motions, a motion capture system, MAC3D [Motion Analysis], was used. There were eight cameras [HMK-200RT; Motion Analysis] employed in this experiment.

Calibration was conducted before the experiment. A Lshape steel bar with four markers was used to decide the absolute coordinate axes of the measurement of the motion capture system and positions of cameras. In addition 500 mm stick wand was employed to improve the accuracy of the measurement in an experimental space; the average wand length was reported as $501.6 \mathrm{~mm}$ and its deviation is 0.70 $\mathrm{mm}$.

Figure 4 shows marker positions to measure a body position in our optical motion capture system. In this experiment, the participant was informed to wear casual dress in order to test if the sensor was utilizable in a daily life. Figure 4-(A) indicates schematic link model used in this study whereas Fig. 4 (B)-(D) show different views (front, back, and side) of actual marker positions attached to the participant. In total, ten markers (M1-M10) were used, and locations of the markers are shown in below.

- M1 \& M2: Right and left shoulder

- M3 \& M4: Right and left back pelvic

- M5 \& M6: Right and left great trchanter

- M7 \& M8: Right and left knee

- M9 \& M10: Right and left ankle

\section{Experimental Task}

In the current study, the accuracy of the developed sensors was tested through two types of motions which are important for medical and rehabilitation: walking, sit-to-stand, and stand-to-sit. Since those two motions are known to be important for daily life [9]; people who lose these motor function would suffer from difficulty in essential mobility for ADL. Also, as described above, the time to perform standing-up and walking (TUG) is used to asses the physical ability. Therefore it was investigated if our gyroscope and accelerometer sensors could measure those two types of motions. In total, there were three trials of walking (Task I-III), and two trials of sit-to-stand motion (Task IV-V).

For the walking trials (Task I-III), a participant was asked to walk forward and backward within a walking area. He started walking from the initial start line toward the target line $(1.7 \mathrm{~m}$ ahead from the start line). Once he reached the target, he walked backward toward the initial place. He kept this motion until he was informed to stop walking.

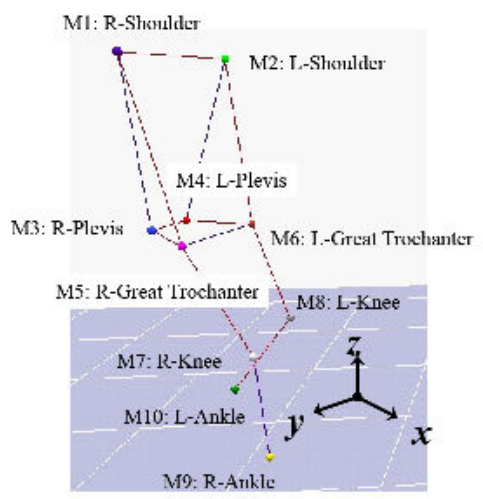

(A)

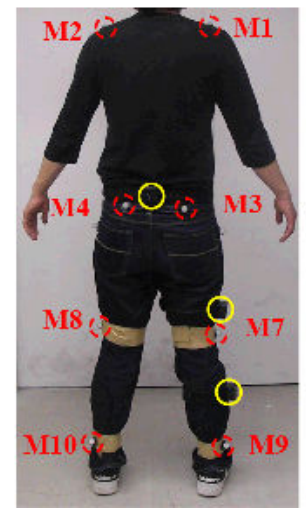

(C)

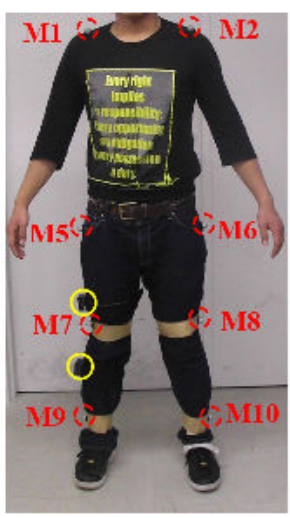

(B)

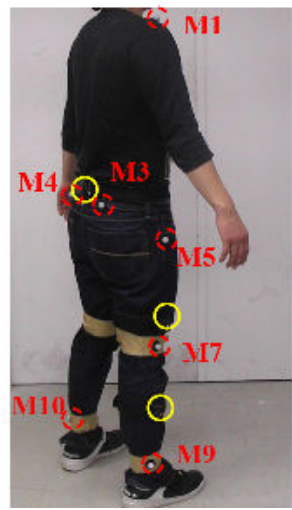

(D)
Fig. 4. (A) A schematic link model used in our motion capture system. Positions and names of markers are also shown. Above pictures (B)-(D) show location of gyroscope and accelerometer sensors (yellow solid circles), and reflective marker positions of the optical motion capture system (red dotted circles).

On the other hand, for the standing-up trials (Task IV-V), the participant was asked to repeat sit-to-stand and stand-tosit motions in the the same place. The duration of the trials were the same as the walking trials; he repeated the motion until the trial finished. In the experiment, the height of the chair was set to $0.45 \mathrm{~m}$.

Calibration was required for our sensors to decided their initial conditions; it took approximately 7-9 s to finish calibration. Therefore, although data recording was $25 \mathrm{~s}$, we asked our participant to perform the motion after the finish of calibration.

From obtained data, start of the motion was decided manually based on the first movement of ankle for the walking task. For the sit-to-stand motion, forward movement of their shoulder was used to decide the start of the motion. The end of the motion was the time when the recording finished. The number of measured motions and their duration times are as follows.

- Task I : 3 forward and 2 backward walking (10.0 s)

- Task II : 3 forward and 2 backward walking (13.5 s)

- Task III: 3 forward and 2 backward walking (13.0 s)

- Task IV : 3 sit-to-stand and 3 stand-to-sit (15.0 s) 
- Task V : 5 sit-to-stand and 5 stand-to-sit $(15.0 \mathrm{~s})$

\section{E. Data Processing}

The sampling rate of data recording was $100 \mathrm{~Hz}$ for both our gyroscope sensors and the motion capture system. Our gyroscope sensors did not employ any filters, and thus each joint angles were computed through simple summation of joint angular velocity as in eq. 1 . In this study, only raw data was used from PocketIMU2 and optical motion capture system.

$$
\theta_{\text {gyro }}^{i=\text { hip,knee,ankle }}(T)=\int_{t=0}^{t=T} \dot{\theta}_{\text {gyro }}^{i=h i p, \text { knee,ankle }}(t) d t
$$

Angles of anterior/posterior inclination were compared between the gyroscope sensors and the motion capture system. From our motion capture system, each body position were recorded as a three dimensional coordinate data.

In order to compute reference inclined joint angles from our motion capture system, Cartesian coordinates were determined based on their pelvic position as in Fig. 5-(A). First, a direction of a $y$-axis was set to the same direction of a vector from right to left great trochanters (M5 and M6). Next, zaxis was determined to the vertical direction to the ground. At last, a direction of a $\mathrm{x}$-axis is decided perpendicularly to $\mathrm{y}$-axis and z-axis.

Joint vectors were considered to calculate inclined joint angles. A hip vector $\left(\mathbf{v}_{\text {hip }}(t)\right)$ at the certain time $t$ was calculated as a cross product of a vector $\left(\mathbf{v}_{\mathrm{M} 5, \mathrm{M} 6}(t)\right)$ between great trochanters (M5-M6) and a vector $\left(\mathbf{v}_{\mathrm{M} 5, \mathrm{M} 3}(t)\right)$ between a right great trochanter (M5) and a right back pelvic (M3) (eq. 2) (Fig. 5-(A)). In the equation, $\mathbf{v}_{\mathrm{i}, \mathrm{j}}$ indicates a vector from $\mathrm{i}$ to $\mathrm{j}$. A knee vector $\left(\mathbf{v}_{\mathrm{knee}}(t)\right)$ was decided based on the direction from the right knee (M7) to the right great trochanter (M5). An ankle vector $\left(\mathbf{v}_{\text {ankle }}(t)\right)$ was decided based on the direction from the right ankle (M9) toward the right knee (M7) as shown in Fig. 5-(A).

$$
\mathbf{v}_{\text {hip }}=\mathbf{v}_{\text {M5,M3 }} \times \mathbf{v}_{\text {M5,M6 }}
$$

In order to calculate angles of anterior/posterior inclination, each vector $\left(\mathbf{v}_{\{\text {ankle,knee,hip\} }}(t)\right)$ was orthographically projected on a sagital plane (x-z plane). An angle between this projected vector $\left.\left(\mathbf{v}^{\prime}{ }_{\{\text {ankle,knee,hip }\}}(t)\right)\right)$ and the z-axis $(\mathbf{z})$ was calculated from eq. 3 . Here, the same Cartesian cooridnates were used to compute ankle and knee joints. In order to compare joint angles from two systems, joint angles when they started the motion was set to 0.0 and changes of angles from the start of each motion were obtained.

$$
\theta_{\mathrm{mc}}^{i=\text { hip,knee, ankle }}=\arccos \left(\frac{\mathbf{v}_{i}^{\prime}(t) \cdot \mathbf{z}}{\left|\mathbf{v}_{i}^{\prime}(t)\right||\mathbf{z}|}\right)
$$

\section{F. Participants}

One healthy man (22 years old) participated in our experiment. Consent was obtained before the experiment started in compliance with the Ethics Committee in the University of Tokyo.

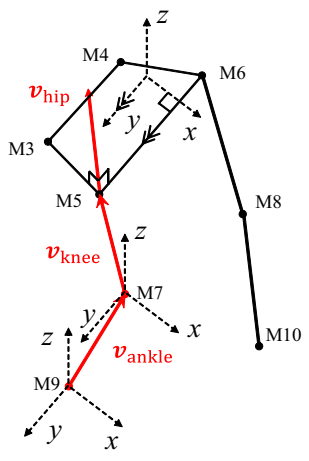

(A)

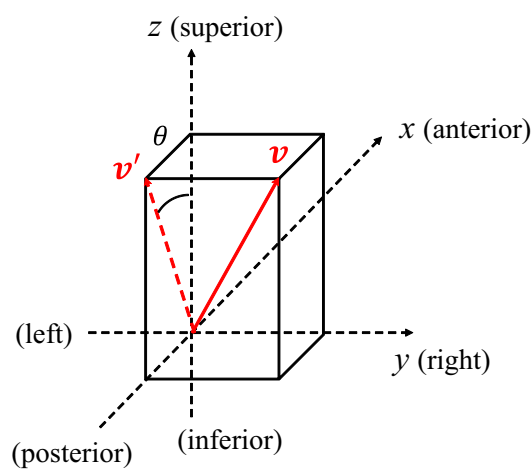

(B)
Fig. 5. (A) Determination of Cartesian coordinates and computation of ankle, knee, and hip joint vectors. (B) Indicates a methodology to calculate inclined joint angles.

\section{G. Evaluation}

Accuracy of our sensors was evaluated by coefficients of correlation and root mean square error (RMSE). Calculated joint angles of our gyroscope sensors $\left(\theta_{\text {gyro }}\right)$ and obtained joint angles from the optical motion capture system $\left(\theta_{m c}\right)$ were compared. Coefficient of correlation was calculated from eq. 4, and root mean square error was calculated from eq. 5 . In the equations, let $\theta_{\text {gyro }}$ be a joint angel computed from our gyroscope sensors, and let $\theta_{m c}$ be a joint angle from the motion capture system. Also, $T_{\text {total }}$ is a total time step of each task.

$$
\begin{gathered}
\frac{\sum_{t=1}^{T_{\text {total }}}\left(\theta_{\text {gyro }}(t)-\theta_{\text {gyro }}(t)\right)\left(\theta_{\mathrm{mc}}(t)-\theta_{\mathrm{mc}}(t)\right)}{\sqrt{\sum_{t=1}^{T_{\text {total }}}\left(\theta_{\mathrm{gyro}}(t)-\theta_{\mathrm{gyro}}(t)\right)^{2}} \sqrt{\sum_{t=1}^{T_{\text {total }}}\left(\theta_{\mathrm{mc}}(t)-\theta_{\mathrm{mc}}(t)\right)^{2}}} \\
R M S E=\sqrt{\frac{\sum_{t=1}^{T_{\text {total }}\left(\theta_{\mathrm{gyro}}(t)-\theta_{\mathrm{mc}}(t)\right)^{2}}}{T_{\text {total }}}}
\end{gathered}
$$

\section{RESULTS}

One healthy man participated in our experiment. In the walking tasks (Task I-III), his walking speed was $0.68 \mathrm{~m} / \mathrm{s}$ $(\mathrm{SE}=0.01)$ for forward walking and $0.58 \mathrm{~m} / \mathrm{s}(\mathrm{SE}<0.00)$ for backward walking. The time to complete sit-to-stand and stand-to-sit were $1.30 \mathrm{~s}(\mathrm{SE}=0.03)$ and $1.37 \mathrm{~s}(\mathrm{SE}=0.03)$ respectively.

Table I shows coefficient of correlation between computed joint angles of PocketIMU2 and our motion capture system. The coefficient of correlation was calculated for ankle, knee, and hip joints of different five tasks (Task I-V). Mean and standard error was also calculated; mean and standard error of coefficient of correlation was $0.986(\mathrm{SE}=0.003)$ for the ankle joint angle, 0.985 ( $\mathrm{SE}=0.004)$ for the knee joint angle, and 0.847 ( $\mathrm{SE}=0.138)$ for the hip joint angle respectively.

Table II shows root mean square error between joint angles of PocketIMU2 and our motion capture system. Mean and standard error was $2.80 \mathrm{deg}(\mathrm{SE}=0.44)$ for the ankle joint 
angle, $3.82 \mathrm{deg}(\mathrm{SE}=0.48)$ for the knee joint angle, and 4.73 ( $\mathrm{SE}=0.48$ ) for the hip joint angle.

Figure 6 shows examples of joint data of PocketIMU and our motion capture. Each column of the figure shows joint angles of the hip, the knee, and the ankle. Three graphs in the top row indicate data from walking motion (Task III), and the bottom three graphs show data from sit-to-stand and stand-to-sit motion (Task V). In the graphs, the blue solid lines indicate the data of PocketIMU and the red dotted lines are from our motion capture system.

In the first row, red plaided boxes indicate a right stance phase in which the participant stood on a single right limb during the walking motion whereas the blue box with diagonal lines show a left stance phase. Areas where both boxes cover indicate the phase when the participants stood on both limbs. In the second row, red dotted box and blue box with vertical lines show two different motions, such as sit-to-stand motion and stand-to-sit motion.

\section{Discussion}

In this study, three joint angles were compared between our gyroscope sensor and the optical motion capture system. Coefficient of correlation and root mean square error were calculated to evaluate the accuracy of the sensor. Different from previous study [5][6], our sensor did not use any filters or make assumption about their posture during motion. Therefore it does not depend on a specific motion, or well organized environment.

We tested the accuracy of the model in the same walking motion and in the same terms, such as coefficient of correlation and root mean square error as the previous study [6]. In terms of RMSE, the current study shows better performance than the previous sutdy; the values for all joints show less than 1.0 in the current study although RSME were between $3.0-4.0$ in the previous study.

TABLE I

COEFFicient of CORRELATION FOR JOINT ANGLES

\begin{tabular}{|c|c|c|c|}
\hline & Ankle Joint & Knee Joint & Hip Joint \\
\hline Task I & 0.987 & 0.983 & 0.548 \\
\hline Task II & 0.988 & 0.984 & 0.664 \\
\hline Task III & 0.984 & 0.971 & 0.678 \\
\hline Task IV & 0.975 & 0.992 & 0.896 \\
\hline Task V & 0.994 & 0.994 & 0.908 \\
\hline \hline Mean & 0.986 & 0.985 & 0.847 \\
\hline SE & 0.003 & 0.004 & 0.138 \\
\hline
\end{tabular}

TABLE II

Root Mean Square ERror for JoInt Angles

\begin{tabular}{|c|c|c|c|}
\hline & Ankle Joint (deg) & Knee Joint (deg) & Hip Joint (deg) \\
\hline Task I & 3.12 & 2.35 & 3.64 \\
\hline Task II & 3.71 & 4.10 & 3.54 \\
\hline Task III & 3.48 & 3.09 & 5.24 \\
\hline Task IV & 2.45 & 4.69 & 5.37 \\
\hline Task V & 1.24 & 4.85 & 5.86 \\
\hline \hline Mean & 2.80 & 3.82 & 4.73 \\
\hline SE & 0.44 & 0.48 & 0.48 \\
\hline
\end{tabular}

Comparing coefficient of correlation, ankle joint shows better performance than that of the previous study (0.99 compared to 0.82), knee joint is about the same (both coefficient of correlation is 0.98 ), but the hip joint shows comparatively lower value ( 0.85 compared to 0.98 ). This can be caused from the sensor location attached on their pelvic. In our experiment, the subject was asked to wear their casual dress in order to test the efficacy in a daily situation. Using a band, sensors on their knee and ankle could be well fixed, and it did not move much with rotation. However, the sensor on the pelvic might be affected from the rotation of their pelvic especially during walking. On the other hand, during Task IV-V (sit-to-stand and stand-to-sit), coefficient of correlation showed better performance since the pelvic inclined but did not rotate much. Despite the lower match of the hip joint, their coefficient of correlation was still enough high to see their movement pattern. The periodic change of joint angles could be observed. Overall, our gyroscope sensor showed adequate accuracy to measure daily movement of human body.

Currently we are developing the new version of gyroscope sensor, called "DIMOTOR" [10]. In this sensor, the accuracy of the sensor will be retained, but the sensor size will be smaller; the height of the sensor will be the $60 \%$ of the current sensor, "PocketIMU2". This enables users to unconcern sensors and move more freely.

In the new system, data of gyroscope and accelerometer is gathered into a data concentrator through RS- 485 connection. Also, the data concentrator can obtain biological data from other devices, such as force plates or surface electromyographic sensors, simultaneously. This concentrator enables easy synchronized data recording with other devices. Obtained data can be sent to a personal computer thorough WiFi connection in spite of bluetooth.

For the further study of our experiment, evaluation of ADL, other than walking and standing-up motion, is warranted. Especially, effect of combination of different motions will be tested since the current experiment only measures the specific motion separately. Also, longer experiment will be needed to test its maximum duration of recording.

\section{COnClusions}

In this paper, accuracy of our developed gyroscope and accelerometer sensor (PocketIMU2) was evaluated in two basic fundamental motions, such as a walking and sit-tostand motion. Ankle, knee and hip joint angles computed from gyroscope sensor were compared to those of optical motion capture system. As a result, coefficients of correlation indicate very high value without any filters. Also, root mean square error shows limited amount of error between gyroscope sensor and motion capture system. The results suggest that our gyroscope and accelerometer sensor can be utilized to measure human joint angles instead of expensive motion capture system. 
Hip Angle

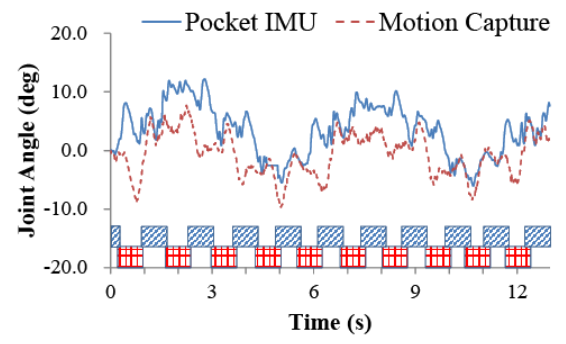

Hip Angle

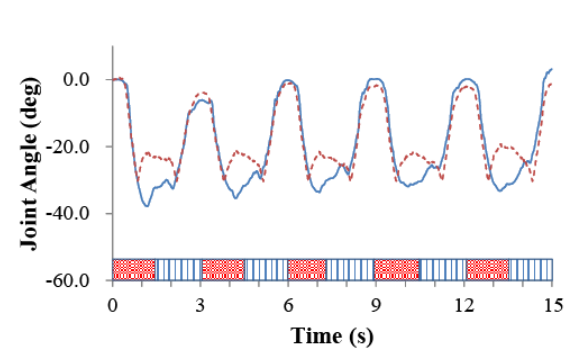

\section{Walking Motion}

Knee Angle

Foot Angle

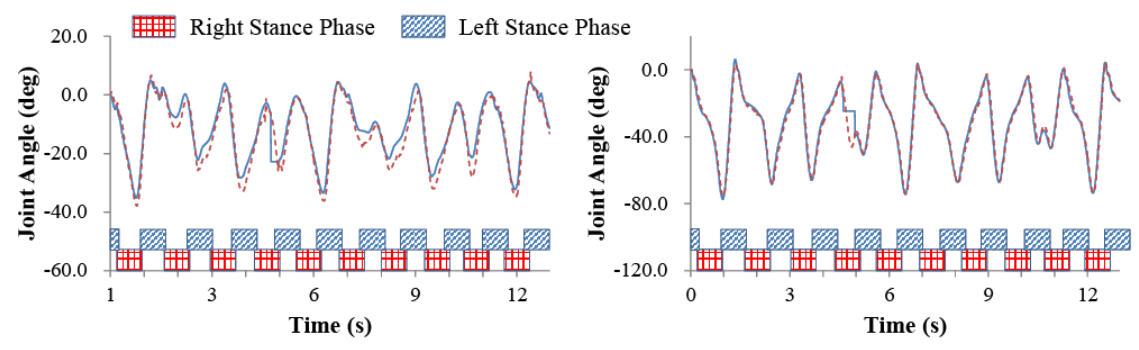

Sit-to-Stand Motion

Knee Angle

Foot Angle
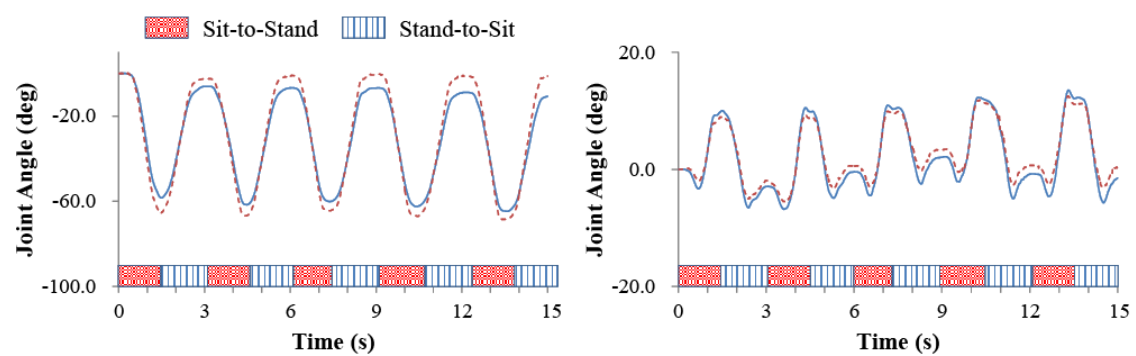

Fig. 6. Above graphs show results of anterior/posterior joint angle of PocketIMU and our motion capture from walking and sit-to-stand motions. Blue solid lines indicate the data of PocketIMU whereas red dotted lines indicate the data of our motion capture system. In the first row, a red plaided box shows a right stance phase, and a box with diagonal lines shows a left stance phase during walking. In the second row, a red dotted box shows a phase of sit-to-stand, and a box with vertical lines indicates a phase of stand-to-sit.

\section{ACKNOWLEDGEMENT}

This work was supported partly by Grant-in-Aid for Scientific Research(B) 24300198.

\section{REFERENCES}

[1] March, L.M. and Bachmeier, C.J.M, "10 Economics of Osteoarthritis: a Global Perspective", Bailliere's Clinical Rheumatology, vol. 11, pp. 817-834, 1997

[2] Bennell, K., Dobson, F., and Hinman, R., "Measures of Physical Performance Assessments: Self-Paced Walk Test (SPWT), Stair Climb Test (SCT), Six-Minute Walk Test (6MWT), Chair Stand Test (CST), Timed Up \& Go (TUG), Sock Test, Lift and Carry Test (LCT), and Car Task", Arthritis Care Res, vol. 63, pp S350-70, 2011

[3] Stone, E.E., and Skubic, M., "Evaluation of an Inexpensive Depth Camera for Passive In-Home Fall Risk Assessment", Proceedings of the International Conference on Pervasive Computing Technologies for Healthcare and Workshop, 2011

[4] Ishigaki, N., Kimura, T., Usui, Y., Aoki, K., Narita, N., Shimizu, M., Hara, K., Ogihara, N., Nakamura, K., kato, H., Ohira, M., Yokokawa, Y., Miyoshi, K., Murakami, N., Okada, S., Nakagmura, T., and Saito, N., "Analysis of Plevic Movement in the Elderly during Walking using a Posture Monitoring System Equipped with a Triaxial Accelerometer and a Gyroscope", Journal of Biomechanics, vol. 44, pp. 1788-1792, 2011

[5] Sagawa, K., Abo, S., Tsukamoto, T., and Kondo, I., "Forearm Trajectory Measurement during Pitching Motion using an Elbow-mounted Sensor", Journal of Advanced Mechanical Design, Systems, and Manufacturing, vol. 74, pp. 400-408, 2009

[6] Watanabe, T., Saito, H., Koike, E., and Nitta, K., "A Preliminary Test of Measurement of Joint Angles and Stride Length with Wireless Inertial Sensors forWearable Gait Evaluation System", Computational Intelligence and Neuroscience, vol. 2011, article ID: 975193, pp. 1-12, 2012
[7] Detailed Information of PocketIMU2 (http: //www. gsport.co.

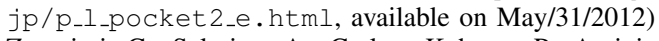

[8] Zampieri, C., Salarian, A., Carlson-Kuhtam, P., Aminian, K., Nutt, J.G., Horak, F.B., “'”The Instrumented Timed Up and Go Test: Potential Outcome Measure for Disease Modifying Therapies in Parkinson's Disease", Journal of Neurology, vol. 81, pp. 171-176, 2009

[9] Guralnik, J.M., Simonsick, E.M., Ferrucci, L., Glynn, R.J., Berkman, L.F., Blazer, D.G., Scherr, P.A., and Wallace, R.B., "A Short Physical Performance Battery Assessing Lower Extremity Function: Association with Self-Reported Disability and Prediction of Mortality and Nursing Home Admission”, Journal of Gerontology, vol. 49, pp. 8594, 1994.

[10] Detailed Information of Dimotor (http://www.gsport.co.jp/ dimotor_e.html available on May/31/2012) 